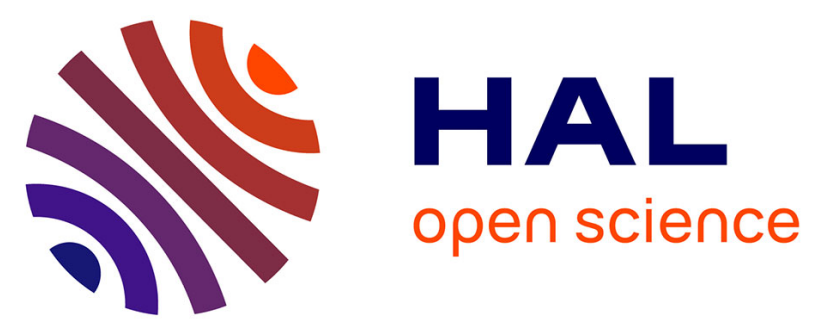

\title{
Temperature-Induced Topological Phase Transition in HgTe Quantum Wells
}

Aleksandr Kadykov, Sergey S. Krishtopenko, Benoit Jouault, Wilfried Desrat, Wojciech Knap, Sandra Ruffenach, Christophe Consejo, Jeremie Torres, S. V. Morozov, N. N. Mikhailov, et al.

\section{To cite this version:}

Aleksandr Kadykov, Sergey S. Krishtopenko, Benoit Jouault, Wilfried Desrat, Wojciech Knap, et al.. Temperature-Induced Topological Phase Transition in HgTe Quantum Wells. Physical Review Letters, 2018, 120 (8), pp.086401. 10.1103/PhysRevLett.120.086401 . hal-01740166

\section{HAL Id: hal-01740166 https://hal.science/hal-01740166}

Submitted on 27 Nov 2018

HAL is a multi-disciplinary open access archive for the deposit and dissemination of scientific research documents, whether they are published or not. The documents may come from teaching and research institutions in France or abroad, or from public or private research centers.
L'archive ouverte pluridisciplinaire HAL, est destinée au dépôt et à la diffusion de documents scientifiques de niveau recherche, publiés ou non, émanant des établissements d'enseignement et de recherche français ou étrangers, des laboratoires publics ou privés. 


\title{
Temperature-Induced Topological Phase Transition in HgTe Quantum Wells
}

\author{
A. M. Kadykov, ${ }^{1,2}$ S. S. Krishtopenko,${ }^{1,2}$ B. Jouault, ${ }^{1}$ W. Desrat, ${ }^{1}$ W. Knap,${ }^{1}$ S. Ruffenach, ${ }^{1}$ C. Consejo, ${ }^{1}$ \\ J. Torres, ${ }^{3}$ S. V. Morozov, ${ }^{2}$ N. N. Mikhailov, ${ }^{4,5}$ S. A. Dvoretskii, ${ }^{4,5}$ and F. Teppe ${ }^{1, *}$ \\ ${ }^{1}$ Laboratoire Charles Coulomb, UMR 5221 Centre National de la Recherche Scientifique, University of Montpellier, \\ F-34095 Montpellier, France \\ ${ }^{2}$ Institute for Physics of Microstructures RAS, GSP-105, Nizhni Novgorod 603950, Russia \\ ${ }^{3}$ Institut d'Electronique et des Systemes, UMR 5214 Centre National de la Recherche Scientifique, University of Montpellier, \\ F-34095 Montpellier, France \\ ${ }^{4}$ Institute of Semiconductor Physics, Siberian Branch, Russian Academy of Sciences, \\ Prospekt Akademika Lavrent'eva 13, 630090 Novosibirsk, Russia \\ ${ }^{5}$ Novosibirsk State University, Pirogova Street 2, 630090 Novosibirsk, Russia
}

(Received 18 October 2017; published 22 February 2018)

\begin{abstract}
We report a direct observation of temperature-induced topological phase transition between the trivial and topological insulator states in an HgTe quantum well. By using a gated Hall bar device, we measure and represent Landau levels in fan charts at different temperatures, and we follow the temperature evolution of a peculiar pair of "zero-mode" Landau levels, which split from the edge of electronlike and holelike subbands. Their crossing at a critical magnetic field $B_{c}$ is a characteristic of inverted band structure in the quantum well. By measuring the temperature dependence of $B_{c}$, we directly extract the critical temperature $T_{c}$ at which the bulk band gap vanishes and the topological phase transition occurs. Above this critical temperature, the opening of a trivial gap is clearly observed.
\end{abstract}

DOI: 10.1103/PhysRevLett.120.086401

The first two-dimensional (2D) systems in which a topological insulator (TI) phase was predicted [1] and then experimentally observed [2] were $\mathrm{HgTe} / \mathrm{Cd}_{x} \mathrm{Hg}_{1-x} \mathrm{Te}$ quantum wells (QWs) with an inverted band structure. The inversion of an electronlike level $E 1$ and a holelike level $H 1$ induces spin-polarized helical edge states [2-4]. When the $E 1$ and $H 1$ levels cross, the band structure mimics a linear dispersion of massless Dirac fermions [5] corresponding to the phase transition between the normal band insulator (NI) and TI states.

The QW thickness $d$ was earlier employed as a tuning parameter of these different quantum phases of matter. The crossing of the energy bands arising when $d$ equals a critical value $d_{c}\left(d_{c} \simeq 6.3 \mathrm{~nm}\right.$ for $x=0.7$ and QWs grown on a CdTe buffer [5]) was measured and identified as a key signature for the topological phase transition [2,5]. However, in addition to the QW thickness, temperature $[6,7]$ and hydrostatic pressure [8] also induce the transition between NI and TI phases across the critical gapless state. The temperature effect on the band ordering in $\mathrm{HgTe} /$ CdHgTe QWs is mainly caused by a strong temperature dependence of the energy gap at the $\Gamma$ point of the Brillouin zone between the $\Gamma_{6}$ and $\Gamma_{8}$ bands in $\mathrm{HgCdTe}$ crystals [9].

Under a magnetic field, a significant way of discriminating the TI phase associated with the helical edge states of the quantum spin Hall effect (QSHE), and the NI phase presenting the chiral edge states of the ordinary quantum Hall effect (QHE), is to probe the behavior of a particular pair of Landau levels (LLs), called zero-mode LLs [5]. These zero-mode LLs split with magnetic field from the $E 1$ and $H 1$ subbands and correspond to the lowest LL of the conduction band and the highest LL of the valence band. In the TI phase, these $E 1$ and $H 1$ subbands are inverted and intersect at the sample boundaries to create the helical edge channels of the QSHE. In this case, the corresponding zero-mode LLs cross at a critical magnetic field $B_{c}$, above which the inverted band ordering is transformed into the normal one [2].

In the NI phase, in which the $E 1$ subband lies above the $H 1$ subband, the zero-mode LLs never cross. This can be conditionally interpreted as negative values for $B_{c}$. Thus, $B_{c}=0$ corresponds to a topological phase transition between NI and TI phases. As it is for the band ordering, a critical magnetic field also depends on temperature and pressure, and therefore can be varied by tuning these external parameters [8].

Recently, Wiedmann et al. [7], by analyzing magnetotransport data at high and low temperatures, have experimentally shown that the TI phase is destroyed at high temperature. However, due to high values of the critical temperature $T_{c}$ (more than $200 \mathrm{~K}$ ) corresponding to the topological phase transition, the presence of a critical gapless state was not directly observed. Another work [10] has reported a temperature evolution of the band gap observed by far-infrared LL spectroscopy. Although the critical temperature was found to be $90 \mathrm{~K}$, an inadequate position of the Fermi level, combined with the inability to 

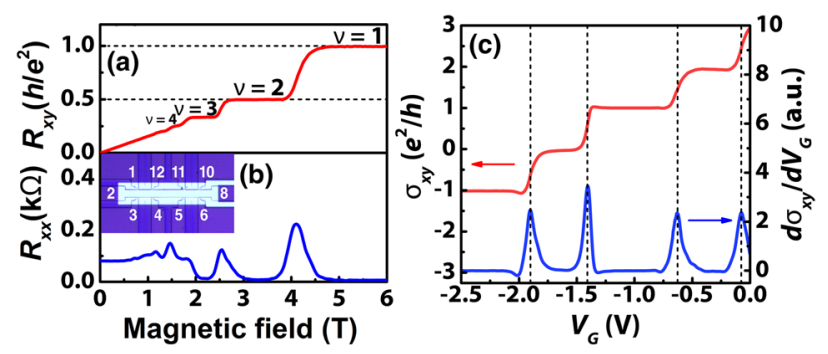

FIG. 1. (a) Hall resistance $R_{x y}$ and (b) $\mathrm{SdH}$ oscillations, $R_{x x}=R_{28,34}$, at $1.7 \mathrm{~K}$ at zero gate voltage. The insert schematically shows a picture of the Hall bar. (c) Hall conductivity $\sigma_{x y}$ and its derivative $\partial \sigma_{x y} / \partial V_{G}$ as a function of gate voltage at $1.7 \mathrm{~K}$ and a magnetic field of $2.4 \mathrm{~T}$.

vary the carrier density in the samples, hindered a clear observation of the topological phase transition.

In this work, we report on the first clear observation of topological phase transition induced by temperature. Accurate values of $B_{c}$ and a temperature phase diagram have been extracted from LL fan charts based on magnetotransport measurements, as firstly performed by Büttner et al. [5] for different QW widths at $4.2 \mathrm{~K}$. By following the temperature dependence of $B_{c}$, we define a critical temperature $T_{c}$, at which the gapless state arises. To describe our experimental results, realistic band structure calculations, based on the eight-band Kane Hamiltonian with temperature-dependent parameters [8], have been performed.

The QW studied in this work was grown by molecular beam epitaxy (MBE) on a [013]-oriented semi-insulating GaAs substrate with a relaxed CdTe buffer [11]. The HgTe QW of $6.5 \mathrm{~nm}$ width was embedded in $40 \mathrm{~nm} \mathrm{Cd}_{0.65} \mathrm{Hg}_{0.35} \mathrm{Te}$ barriers (critical thickness $d_{c}=6.2 \mathrm{~nm}$; see also Fig. 4). A $40 \mathrm{~nm}$ CdTe cap layer was deposited on top of the structures. The barriers from both sides of the QW were selectively doped with indium, resulting in a $2 \mathrm{D}$ electron concentration of a few $10^{11} \mathrm{~cm}^{-2}$ at low temperatures. After MBE growth, $100 \mathrm{~nm} \mathrm{SiO}{ }_{2}$ and $200 \mathrm{~nm} \mathrm{Si}_{3} \mathrm{~N}_{4}$ dielectric layers were deposited on top of the structure by a plasmochemical method. The gated Hall bar has a total length of $650 \mu \mathrm{m}$ and a total width of $50 \mu \mathrm{m}$. Magnetotransport measurements have been performed in a variable temperature insert with a base temperature $T=1.7 \mathrm{~K}$ equipped with a superconducting coil. All the measurements have been done with a current of $1 \mathrm{nA}$, to avoid heating.

Figures 1(a) and 1(b) present Hall resistance and Shubnikov-de Haas $(\mathrm{SdH})$ oscillations at $1.7 \mathrm{~K}$. The Hall resistance shows pronounced plateaus at both even and odd multiples of $h / e^{2}$. The Hall conductivity as a function of the gate voltage $V_{G}$ at $1.7 \mathrm{~K}$ and a magnetic field of $2.4 \mathrm{~T}$, as well as its derivative $\partial \sigma_{x y} / \partial V_{G}$, are both shown in Fig. 1(c). Each peak corresponds to the crossings of the Fermi level with the given LL. This method allows for a visualization of LLs from $E 1$ and $H 1$ subbands and clear reconstruction of their behavior in magnetic fields.

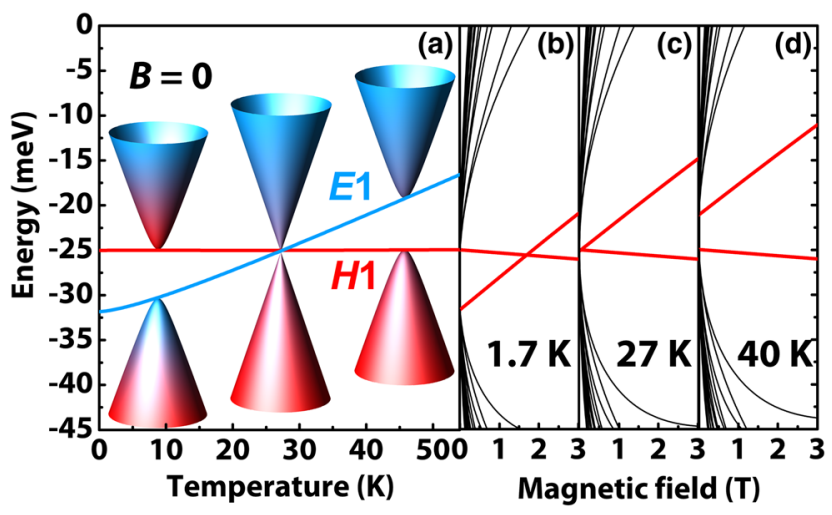

FIG. 2. (a) Evolution of $E 1$ and $H 1$ subbands (at $\mathbf{k}=0$ ) and band structure in the sample with temperature. Blue and red shading indicate contributions from electronlike and holelike states, respectively, at a given quasimomentum $\mathbf{k}$ point. (b)-(d) LL fan chart at different temperatures: (b) $1.7 \mathrm{~K}$ (TI phase), (c) $27 \mathrm{~K}$ (critical gapless state), and (d) $40 \mathrm{~K}$ (NI phase). A pair of zero-mode LLs is presented by red curves.

Figure 2(a) provides a temperature evolution of $E 1$ and $H 1$ subbands at zero quasimomentum, and Figs. 2(b)-2(d) show the corresponding LL fan charts at three different temperatures, evidencing the position of the zero-mode LLs. The upper zero-mode LL level split from the conduction band has a pure heavy-hole character, and its energy decreases with $B$. By contrast, the second zeromode LL starting from the valence band has an electron component [2], and its energy increases with $B$.

At $T \simeq 1.7 \mathrm{~K}$, the $E 1$ subband is well below the $H 1$ subband, and the two corresponding zero-mode LLs cross at a positive magnetic field value $B_{c} \simeq 1.6 \mathrm{~T}$. This positive $B_{c}$ characterizes the TI phase. By increasing the temperature, the width of the TI gap decreases, yielding a $B_{c}$ that decreases. At a critical temperature $T_{c}=27 \mathrm{~K}$, the zeromode LLs intersect at $B_{c} \simeq 0 \mathrm{~T}$, which means that the subbands $E 1$ and $H 1$ have the same energy, and therefore the topological gap closes. At $T_{c}$, the system hosts singlevalley massless Dirac fermions [5]. Above $T_{c}$, the crossing of the zero-mode LLs can be extrapolated to a negative value of magnetic field.

To demonstrate experimentally a temperature-driven phase transition in our samples, we reconstruct the LL fan chart by means of magnetotransport, measured in a wide range of gate voltages at temperatures from $1.7 \mathrm{~K}$ up to $40 \mathrm{~K}$. As mentioned above, the derivative of the Hall conductivity has its maximum values when the Fermi level crosses one of the LLs. Therefore, plotting $\partial \sigma_{x y} / \partial V_{G}$ for each magnetic field value makes it possible to visualize the LL fan chart [5]. This allows for accurate extraction of the critical magnetic field $B_{c}$, at which the zero-mode LLs cross. Since $B_{c}$ is related to the changing of band ordering, the measurement of LL fan charts performed by tuning the temperature is an efficient tool to probe a temperatureinduced phase transition between NI and TI phases. 


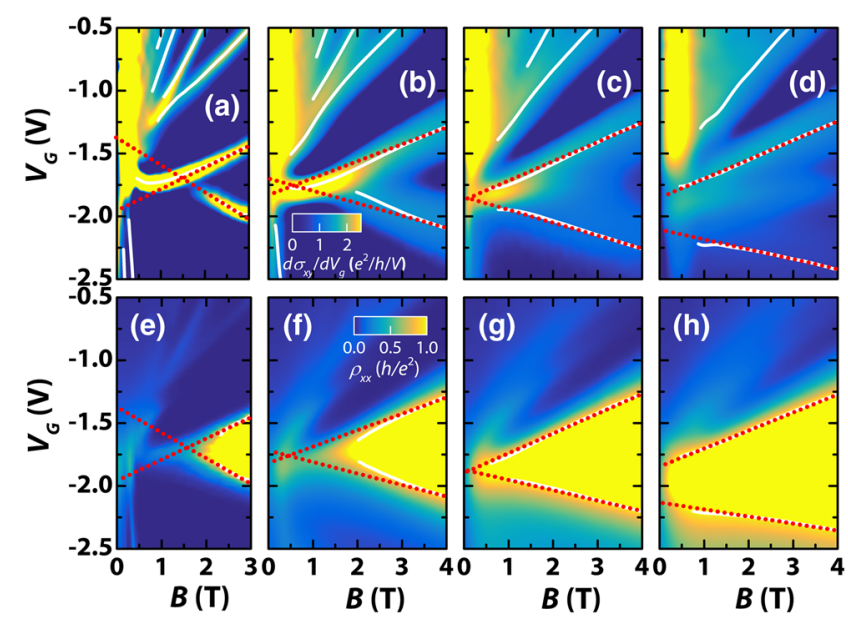

FIG. 3. (a)-(d) Color map of $\partial \sigma_{x y} / \partial V_{G}$ as a function of both magnetic field and gate voltage, at (a) $1.7 \mathrm{~K}$, (b) $20 \mathrm{~K}$, (c) $27 \mathrm{~K}$, and (d) $40 \mathrm{~K}$. The white solid curves correspond to $\sigma_{x y}=$ $(n+1 / 2) e^{2} / h$. In the NI state $(B>2.2 \mathrm{~T})$, the isolines of $\sigma_{x y}=$ $\pm e^{2} / 2 h$ are linearly fitted by the red dotted lines giving the values of $B_{c}$. (e)-(h) The false-color maps show the longitudinal resistivity at the same temperatures as in the respective top panels. The white curves correspond to the isolines of $\rho_{x x}=h / e^{2}$. The red dotted lines are the linear fits of these curves in the field range of $B=2.2-6 \mathrm{~T}$. The crossing point of the two red lines gives another estimate for $B_{c}$.

The color maps in Figs. 3(a)-3(d) were obtained by plotting the derivative $\partial \sigma_{x y} / \partial V_{G}$ as a function of $B$ at different temperatures. The two zero-mode LLs are observed separately above $B \simeq 1.5 \mathrm{~T}$. They seem to emerge from the resistance maximum at $V_{g}=-1.7 \mathrm{~V}, B=0 \mathrm{~T}$. The $\partial \sigma_{x y} / \partial V_{G}$ trace of the zero-mode LL, originating from the $H 1$ subband, obviously broadens and fades out at large $T$, probably due to a strong temperature dependence of mobility caused by the large effective mass of holes. It becomes hardly distinguishable above $20 \mathrm{~K}$. However, the zero-mode LLs also coincide with $\sigma_{x y}=$ $\pm e^{2} / 2 h$ and $\rho_{x x} \simeq h / e^{2}$ [from the semicircle relation $\sigma_{x x}^{2}+\left(\sigma_{x y} \mp 1 / 2\right)^{2}=1 / 4$; see Ref. [12]].

In Figs. 3(a)-3(d), the white curves correspond to $\sigma_{x y}=(n+1 / 2) e^{2} / h$, where $n$ is an integer. They are clearly defined up to $40 \mathrm{~K}$ and underline the LL positions, in agreement with the $\partial \sigma_{x y} / \partial V_{G}$ maxima. The estimated positions of the two zero-mode LLs do not follow the lines of constant filling factor, which implies that LLs overlap. Numerical simulations [13], performed by taking into account a LL-broadening $\Gamma \simeq 4 \mathrm{meV}$ provide evidence that $B_{c}$ can be estimated from the linear extrapolation of the LL position in the NI gap, toward lower magnetic fields. The linear interpolations of the two curves $\sigma_{x y}= \pm e^{2} / 2 h$ for $B>2.2 \mathrm{~T}$ are marked by the red dotted lines. At $T=1.7 \mathrm{~K}$, these lines cross at a finite magnetic field and give an estimate $B_{c} \approx 1.5 \mathrm{~T}$. As $T$ increases, the crossing goes to lower magnetic fields, and it finally vanishes at $T \simeq 27 \mathrm{~K}$. The crossing of the zero-mode LLs at zero magnetic field gives a direct indication of a critical gapless state [5], revealing a topological phase transition at $T \simeq 27 \mathrm{~K}$. At higher temperatures $[T=40 \mathrm{~K}$; see Fig. 3(d)], the crossing of the zero-mode LLs can be extrapolated to a negative value of magnetic field, evidencing a NI phase with trivial band ordering.

To check the last point, we also plot the longitudinal resistivity in color-coded graphs as a function of $B$ and $V_{G}$ at temperatures up to $40 \mathrm{~K}$; see the bottom panels in Fig. 3 . The whole set of LLs is not seen here as in the top panels, but the traces of both zero-mode LLs are clearly visible at the edges of the main $\rho_{x x}$ peak. The red dotted lines correspond to the linear fitting of the isolines of $\rho_{x x}=h / e^{2}$ in the field range of $B=2.2-6 \mathrm{~T}$. The crossing of the fitting lines in lower fields gives another estimate of $B_{c}$.

Figure 4 provides a comparison of experimental and theoretical values of the critical magnetic field. Details of theoretical calculations can be found elsewhere [8]. Additional analysis based on a simplified Dirac-like Hamiltonian shows that the temperature evolution of $B_{c}$ is caused by the temperature dependence of the band gap in our sample (see Fig. 2), while the temperature effect on the dispersion of the zero-mode LLs is negligibly small [13]. Two sets of experimental values have been obtained from the analysis of experimental data presented in the top and bottom panels of Fig. 3. For each set, the experimental precision for $B_{c}$ is the $(1 \sigma)$ standard deviation given by the $\rho_{x x}$ and $\sigma_{x y}$ estimates. It is seen that experimental values of $B_{c}$ from different sets reproduce quantitatively the same temperature dependence of the critical magnetic field and

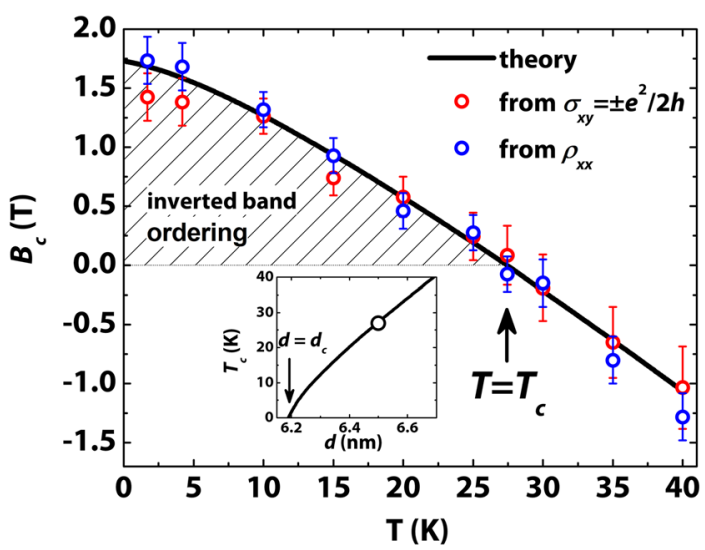

FIG. 4. Theoretical (solid curve) and experimental (open symbols) values of the critical magnetic field $B_{c}$ as a function of temperature. The values marked by the red symbols are obtained from the data set in the top panels of Fig. 3. The blue symbols mark the values of $B_{c}$ extracted from $\rho_{x x}$. The sparse and white regions correspond to the inverted and trivial band ordering, respectively. The inset shows $T_{c}$ as a function of $d$ in (013) $\mathrm{HgTe} / \mathrm{Cd}_{0.65} \mathrm{Hg}_{0.35} \mathrm{Te} \mathrm{QWs}$ grown on a $\mathrm{CdTe}$ buffer. The open symbol represents the experimental value measured in our sample. 
are in good agreement with the theoretical values at all the temperatures. The critical magnetic field vanishes at $T_{c} \simeq 27 \mathrm{~K}$, which corresponds to the formation of gapless states, in which the system mimics massless Dirac fermions [5]. We formally extend our comparison toward negative values of $B_{c}$, which correspond to the formation of the NI state. Note that the error in the determination of $B_{c}$ from $\sigma_{x y}= \pm e^{2} / 2 h$ increases with temperature due to broadening of the trace of the zero-mode LL, originating from the $H 1$ subband as it is discussed above. Thus, our results shown in Fig. 4 are eloquent proof of the temperatureinduced topological transition in HgTe QWs.

We note that the size of the Hall bar in our sample largely exceeds the typical spin relaxation length $(\sim 1 \mu \mathrm{m})$ [20]. An additional four probes' resistance measurements (see Sec. E in the Supplemental Material [13]) provide evidence that although the edge states exist at $B<B_{c}$ $[21,22]$, they do not contribute significantly to the conductivity of our sample.

Finally, we discuss the role of spin-orbital corrections resulting from bulk inversion asymmetry (BIA) [23] of zincblend crystals and interface inversion asymmetry (IIA) [24] on the behavior of zero-mode LLs. Both reasons induce the anticrossing of zero-mode LLs in the vicinity of $B_{c}$. So far, the fingerprint of such anticrossing was observed only by far-infrared LL spectroscopy [10,25-27] focused on LL transitions involving the zero-mode LLs.

We note that spin-orbital corrections resulting from structure inversion asymmetry (SIA) of the QW profile do not lead to anticrossing behavior of zero-mode LLs. Moreover, since we do not observe any beatings of $\mathrm{SdH}$ oscillations induced by SIA at the gate voltages presented in Fig. 3, we conclude that SIA-induced corrections are small in our sample. The latter is also confirmed by good agreement between our experimental data and calculations performed for the symmetrical QW profile.

The linear dependence of energies of the zero-mode LLs and their experimental traces on magnetic field far from $B_{c}$ enables us to recalculate the anticrossing gap $\Delta$ measured in magneto-optics [25-27] into the values of gate voltage expected in our sample. For instance, by using a theoretical band gap of $7 \mathrm{meV}$ at $1.7 \mathrm{~K}$ and $B=3 \mathrm{~T}$ (see Fig. 2) and its experimental value $\Delta V_{G} \simeq 0.7 \mathrm{~V}$ represented in the scale of $V_{g}$ [see Fig. 3(e)], we find that the experimental values of $\Delta \simeq 4-5 \mathrm{meV}$ transform into $0.4-0.5 \mathrm{~V}$, which should be observed for our sample. As is seen from Fig. 3, the width of experimental traces of zero-mode LLs in the vicinity of $B_{c}$, which may be interpreted as a manifestation of the LL anticrossing, is significantly lower than the mentioned values. The latter is also consistent with previous magnetotransport results $[2,5]$.

As discussed in Ref. [28], a possible explanation of the large anticrossing gap in the vicinity of $B_{c}$ measured by farinfrared LL spectroscopy can be based on electron-electron interaction effects. The latter may largely influence close energies of LL transitions [29-31] as we deal with strongly nonparabolic 2D systems for which Kohn's theorem [32] does not hold.

On the other hand, both BIA and IIA induce a spin splitting of both electronlike and holelike states at nonzero $\mathbf{k}$ in the symmetrical QWs. If the spin splitting is strong enough, it results in the beatings arising in $\mathrm{SdH}$ oscillations. However, these beatings have never been observed in symmetrical $\mathrm{HgTe}$ QWs at low electron concentration. This is also consistent with the small strength of BIA and IIA terms, evaluated for our sample.

Finally, the presence of BIA and IIA terms induces the optical transitions between two branches of helical edge states [33]. If both terms are small, only spin-dependent transitions between edge and bulk states are allowed [34]. Very recent accurate measurements of a circular photogalvanic current in HgTe QWs [35] have revealed the optical transitions between the edge and bulk states only. The latter also indicates the small effects of BIA and IIA terms in $\mathrm{HgTe}$ QWs.

In conclusion, we have directly observed a temperatureinduced topological transition between NI and TI phases. By plotting experimental LL fan charts at different temperatures, we have accurately extracted values of critical magnetic field at different temperatures. Following the experimental phase diagram, we have determined a critical temperature $T_{c} \simeq 27 \mathrm{~K}$, at which $B_{c}$ vanishes. Our experimental results are in good agreement with realistic band structure and LL calculations based on the eight-band Kane Hamiltonian with temperature-dependent parameters.

The authors gratefully thank Z. D. Kvon from the Institute of Semiconductor Physics (Siberian Branch, Russian Academy of Sciences) for processing of the samples. Part of this work was supported by the Languedoc-Roussillon region via the "Gepeto Terahertz platform," by the Era.NetRus Plus project "Terasens," by the CNRS through "Emergence project 2016" and LIA "TeraMIR," by MIPS department of Montpellier University through the "Occitanie Terahertz Platform" and the ARPE project "Terasens," and by the French Agence Nationale pour la Recherche (Grant No. ANR-16-CE09-0016 and the Dirac3D project). Theoretical calculations were performed in the framework of Project No. 16-12-10317 provided by the Russian Science Foundation. The growth of the samples were supported by the Russian Foundation for Basic Research (Grants No. 1552-16017 and No. 18-52-16008). S. S. K. and A. M. K. acknowledge the Russian Ministry of Education and Science (MK-1136.2017.2 and SP-5051.2018.5).

S. S. K. and B. J. contributed equally to this work.

*frederic.teppe@umontpellier.fr

[1] B. A. Bernevig, T. L. Hughes, and S.-C. Zhang, Science 314, 1757 (2006). 
[2] M. König, S. Wiedmann, C. Brüne, A. Roth, H. Buhmann, L. W. Molenkamp, X.-L. Qi, and S.-C. Zhang, Science 318, 766 (2007).

[3] A. Roth, C. Brüne, H. Buhmann, L. W. Molenkamp, J. Maciejko, X.-L. Qi, and S.-C. Zhang, Science 325, 294 (2009).

[4] C. Brüne, A. Roth, H. Buhmann, E. Hankiewicz, L. Molenkamp, J. Maciejko, Q. X. -L., and S.-C. Zhang, Nat. Phys. 8, 485 (2012).

[5] B. Büttner, C. Liu, G. Tkachov, E. Novik, C. Brüne, H. Buhmann, E. Hankiewicz, P. Recher, B. Trauzettel, S. Zhang, and L. Molenkamp, Nat. Phys. 7, 418 (2011).

[6] P. Sengupta, T. Kubis, Y. Tan, M. Povolotskyi, and G. Klimeck, J. Appl. Phys. 114, 043702 (2013).

[7] S. Wiedmann, A. Jost, C. Thienel, C. Brüne, P. Leubner, H. Buhmann, L. W. Molenkamp, J. C. Maan, and U. Zeitler, Phys. Rev. B 91, 205311 (2015).

[8] S. S. Krishtopenko, I. Yahniuk, D. B. But, V. I. Gavrilenko, W. Knap, and F. Teppe, Phys. Rev. B 94, 245402 (2016).

[9] F. Teppe, M. Marcinkiewicz, S. S. Krishtopenko, S. Ruffenach, C. Consejo, A. M. Kadykov, W. Desrat, D. But, W. Knap, J. Ludwig, S. Moon, D. Smirnov, M. Orlita, Z. Jiang, S. V. Morozov, V. Gavrilenko, N. N. Mikhailov, and S. A. Dvoretskii, Nat. Commun. 7, 12576 (2016).

[10] M. Marcinkiewicz, S. Ruffenach, S. S. Krishtopenko, A. M. Kadykov, C. Consejo, D. B. But, W. Desrat, W. Knap, J. Torres, A. V. Ikonnikov, K. E. Spirin, S. V. Morozov, V. I. Gavrilenko, N. N. Mikhailov, S. A. Dvoretskii, and F. Teppe, Phys. Rev. B 96, 035405 (2017).

[11] S. Dvoretsky, N. Mikhailov, Y. Sidorov, V. Shvets, S. Danilov, B. Wittman, and S. Ganichev, J. Electron. Mater. 39, 918 (2010).

[12] D. Shahar, D. C. Tsui, M. Shayegan, E. Shimshoni, and S. L. Sondhi, Phys. Rev. Lett. 79, 479 (1997).

[13] See the Supplemental Material at http://link.aps.org/ supplemental/10.1103/PhysRevLett.120.086401, which also contains Refs. [14-19], for a brief discussion of the joint effects of disorder and geometric capacitance of the gated Hall bar on experimental Landau level fan charts. Analysis of the temperature dependence of the critical magnetic field is also provided therein.

[14] A. M. Dykhne and I. M. Ruzin, Phys. Rev. B 50, 2369 (1994).

[15] D. G. Polyakov and B. I. Shklovskii, Phys. Rev. Lett. 70, 3796 (1993).

[16] K. Bennaceur, P. Jacques, F. Portier, P. Roche, and D. C. Glattli, Phys. Rev. B 86, 085433 (2012).

[17] A. A. Abrikosov, Phys. Rev. B 58, 2788 (1998).
[18] S. S. Krishtopenko, W. Knap, and F. Teppe, Sci. Rep. 6, 30755 (2016)

[19] D. Shahar, D. C. Tsui, M. Shayegan, E. Shimshoni, and S. L. Sondhi, Phys. Rev. Lett. 79, 479 (1997).

[20] G. Grabecki, J. Wróbel, M. Czapkiewicz, L. Cywiński, S. Gierałtowska, E. Guziewicz, M. Zholudev, V. Gavrilenko, N. N. Mikhailov, S. A. Dvoretski, F. Teppe, W. Knap, and T. Dietl, Phys. Rev. B 88, 165309 (2013).

[21] G. Tkachov and E. M. Hankiewicz, Phys. Rev. Lett. 104, 166803 (2010).

[22] J.-C. Chen, J. Wang, and Q.-F. Sun, Phys. Rev. B 85, 125401 (2012).

[23] M. König, H. Buhmann, L. W. Molenkamp, T. Hughes, C.-X. Liu, X.-L. Qi, and S.-C. Zhang, J. Phys. Soc. Jpn. 77, 031007 (2008).

[24] M. V. Durnev and S. A. Tarasenko, Phys. Rev. B 93, 075434 (2016).

[25] M. Orlita, K. Masztalerz, C. Faugeras, M. Potemski, E. G. Novik, C. Brüne, H. Buhmann, and L. W. Molenkamp, Phys. Rev. B 83, 115307 (2011).

[26] M. Zholudev, F. Teppe, M. Orlita, C. Consejo, J. Torres, N. Dyakonova, M. Czapkiewicz, J. Wróbel, G. Grabecki, N. Mikhailov, S. Dvoretskii, A. Ikonnikov, K. Spirin, V. Aleshkin, V. Gavrilenko, and W. Knap, Phys. Rev. B 86, 205420 (2012).

[27] M. S. Zholudev, F. Teppe, S. V. Morozov, M. Orlita, C. Consejo, S. Ruffenach, W. Knap, V. I. Gavrilenko, S. A. Dvoretskii, and N. N. Mikhailov, JETP Lett. 100, 790 (2015).

[28] A. M. Kadykov, J. Torres, S. S. Krishtopenko, C. Consejo, S. Ruffenach, M. Marcinkiewicz, D. But, W. Knap, S. V. Morozov, V. I. Gavrilenko, N. N. Mikhailov, S. A. Dvoretsky, and F. Teppe, Appl. Phys. Lett. 108, 262102 (2016).

[29] A. H. MacDonald and C. Kallin, Phys. Rev. B 40, 5795 (1989).

[30] Y. A. Bychkov and G. Martinez, Phys. Rev. B 72, 195328 (2005).

[31] S. S. Krishtopenko, A. V. Ikonnikov, M. Orlita, Y. G. Sadofyev, M. Goiran, F. Teppe, W. Knap, and V. I. Gavrilenko, J. Appl. Phys. 117, 112813 (2015).

[32] W. Kohn, Phys. Rev. 123, 1242 (1961).

[33] M. V. Durnev and S. A. Tarasenko (to be published).

[34] V. Kaladzhyan, P. P. Aseev, and S. N. Artemenko, Phys. Rev. B 92, 155424 (2015).

[35] K.-M. Dantscher, D. A. Kozlov, M. T. Scherr, S. Gebert, J. Bärenfänger, M. V. Durnev, S. A. Tarasenko, V. V. Bel'kov, N. N. Mikhailov, S. A. Dvoretsky, Z. D. Kvon, J. Ziegler, D. Weiss, and S. D. Ganichev, Phys. Rev. B 95, 201103 (2017). 